\title{
202 In the cavity of the larynx
}

A The rima glottidis is the interval between the false cords.

B Reinke's space lies between the surface epithelium and the deeper elastic layer.

C Keratinizing stratified squamous epithelium lines the true cords.

D The posterior part of the ventricular sinus contains the mucus secreting saccule.

E The paraglottic and pre-epiglottic spaces are continuous.

\section{Neurovascular supply and lymphatic drainage of the larynx}

A The main blood supply is from branches of the superior and inferior thyroid arteries.

B Lymph from the supraglottic larynx drains to the pre-epiglottic and upper deep cervical nodes.

C The internal branch of the superior laryngeal nerve is entirely motor.

D The external branch of the superior laryngeal nerve supplies the cricothyroid muscle.

E The recurrent laryngeal nerve is sensory above the true cords.

\section{Examination of the larynx}

A The anterior commissure is easily visualized on indirect laryngoscopy during quiet inspiration.

B The Negus laryngoscopes have distal illumination.

C Stroboscopy is of most value in studying vocal cords during quiet respiration.

D Anteroposterior tomography is valuable in assessing the degree of spread of subglottic carcinoma.

E A grating sensation on moving the larynx from left to right is abnormal. 